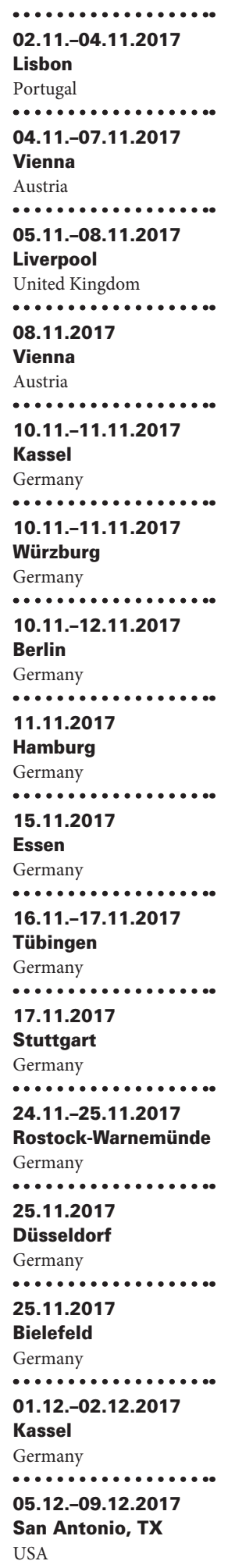

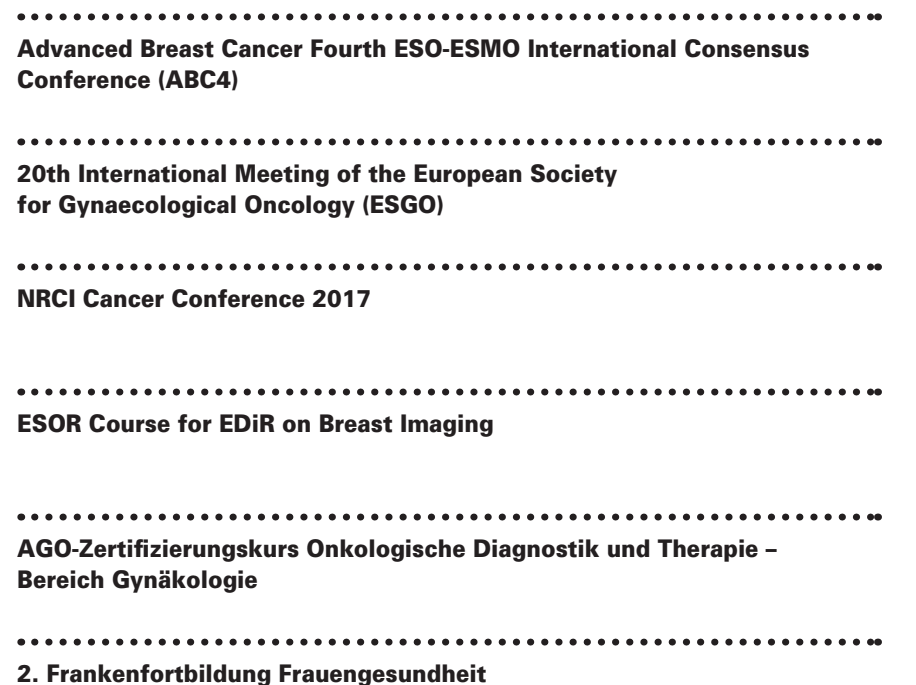

\section{Frankenfortbildung Frauengesundheit}

8. Chefärzte-Jahrestagung der Bundesarbeitsgemeinschaft Leitender Ärztinnen und Ärzte in der Frauenheilkunde und Geburtshilfe e.V. (BLFG)

Hamburger Intensivseminar GynOnko

Best of Europe. State of the Art bei soliden Tumoren -

Aktuelles vom Europäischen Krebskongress

7. Tübinger Brustchirurgische Tage Studiengruppe

20. Ostsee-Seminar 2017 - Frauenheilkunde und Geburtshilfe

\section{Rhein-Ruhr-Symposium}

GynOnko-Kongress

AGO-Zertifizierungskurs Onkologische Diagnostik und Therapie Bereich Gynäkologie

San Antonio Breast Cancer Symposium
Information: www.abc-lisbon.org

Information: www.esgo.org/attend/congress

Information: www.conference.ncri.org.uk

Information: www.esor.org

Information: www.medconcept.org

Information: www.if-kongress.de/veranstaltungen/ 2017/2-franken-fortbildung-frauengesundheit/

Information: www.if-kongress.de/veranstaltungen

Information: www.eickeler.org/HIGO.pdf

Information: www.eickeler.org/BoE.pdf

Information: www.if-kongress.de/veranstaltungen

Information: www.ago-ovar.de

Information: www.if-kongress.de/veranstaltungen

Information: www.rhein-ruhr-symposium.de

Information: www.karriere.evkb.de/

fort-und-weiterbildung/fortbildung/fortbildung-details/ artikel//gynoaekologie-und-onkologie-kongress.html

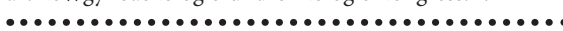
Information: www.medconcept.org

Information: www.sabcs.org

\section{KARGER}

Fax +497614520714

Information@Karger.com

www.karger.com

\section{๑) 2017 S. Karger GmbH, Freiburg}




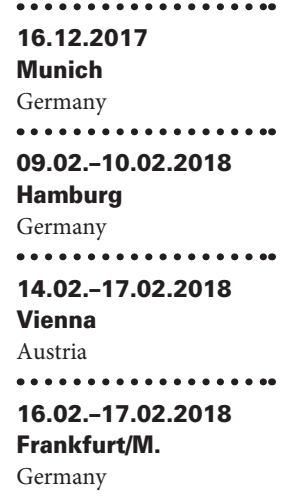

21.0..............

Berlin

Germany

•..............

24.02.-25.02.2018

Wiesbaden

Germany

... . . . . . . . . .

Frankfurt/M.

Germany

................

08.03.-11.03.2018

Miami, FL

USA

09.0...............

Kassel

Germany

•....................

09.03.-11.03.2018

Nuremberg

Germany

10.03.2018

Frankfurt/M

Germany

................

17.03.2018

Essen

Germany

...............

21.03.-23.03.2018

Barcelona

Spain

14.04 .2018

Frankfurt/M.

Germany

•...............

20.04.-21.04.2018

Mainz

Germany

03.05 .2018

Lucerne

Switzerland

•..............

04.05.-05.05.2018

Kassel

Germany

[....................

Kampen/Sylt

Germany

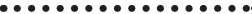

01.06.-05.06.2018

Chicago, IL

USA
XI. Wissenschaftliches Wintersymposium

Norddeutsches Onkologieforum

ECCO 2018 - European Cancer Congress

10. Kongress Gynäkologie und Geburtshilfe Frankfurt 2018

33. Deutscher Krebskongress - Perspektiven verändern Krebs -

Krebs verändert Perspektiven. Diagnose, Therapie, (Über-)Leben

19. Symposium Mammasonographie

Jahrestreffen der German Breast Group (GBG)

35th Annual Miami Breast Cancer Conference

AGO-Zertifizierungskurs Diagnostik und Therapie

in der onkologischen Gynäkologie

23. Internationaler Fortbildungskurs Moderne Mammadiagnostik und -therapie - Standards, Trends, Perspektiven

AGO-Mamma State of the Art 2018

8. Essener Symposium zur gynäkologischen Onkologie und Senologie

11th European Breast Cancer Conference (EBCC)

AGO Aktuell - 2018

12. Gyn-Onko-Update

5. Luzerner Symposium für Gynäkologische Onkologie

AGO-Zertifizierungskurs Diagnostik und Therapie

in der onkologischen Gynäkologie

Sylter Fortbildungstage Frauenheilkunde und Gynäkologie

Asco Annual Meeting
Information: www.wintersymposium-muenchen.de

Information: www.if-kongress.de/veranstaltungen

Information: www.ecco-ibd.eu/eccols

Information: www.cocs.de

\section{Information:}

www.krebsgesellschaft.de/deutsche-krebsgesellschaft/ konferenzen/deutscher-krebskongress.html

Information: www.comed-kongresse.de/kongresse/ 19-symposium-mammasonographie

Information: www.gbg.de

Information: www.eventsinamerica.com/events/ 35th-annual-miami-breast-cancer-conference-2018/ ev56a6914c9bf26/

Information: www.medconcept.org

Information: www.comed-kongresse.de

Information: www.ago-online.de/de/veranstaltungen

Information: info@eickeler.org

Information: www.ecco-org.eu/Events/EBCC11

Information: www.ago-online.de/de/veranstaltungen

Information: www.gyn-onko-update.com

Information: www.gyn-onko-luzern.ch

Information: www.medconcept.org

Information: www.gynsylt.de

Information: https://am.asco.org 
06.06.-09.06.2018

Vancouver, BC

Canada

14.06.-16.06.2018

Stuttgart

Germany

...............

05.09.-08.09.2018

Bremen

Germany

…....

07.09.-09.09.2018

Vienna

Austria

14. . . . . . . . .

Kyoto

Japan

•.............

28.09.-02.10.2018

Vienna

Austria

A..................

30.10.-03.11.2018

Berlin

Germany

...............

11.10.-13.10.2018

Istanbul

Turkey

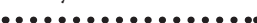

19.10.-23.10.2018

Munich

Germany

•...............

31.10.-03.11.2018

Berlin

Germany

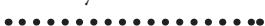

03.04.-06.04.2019

Berlin

Germany 16th World Congress on Menopause - Midlife Health in the 21st Century

38. Jahrestagung der Deutschen Gesellschaft für Senologie

12. Kongress der Deutschen Gesellschaft für Palliativmedizin

ECCO 2018: European Cancer Summit

17th Biennial Meeting of the International Gynecologic Cancer Society

Jahrestagung der Deutschen, Österreichischen und Schweizerischen Gesellschaften für Hämatologie und Medizinische Onkologie

62. Kongress der Deutschen Gesellschaft für Gynäkologie und

Geburtshilfe

3rd Breast Cancer Conference. Breastanbul

ESMO 2018 Congress

62. Kongress der Deutschen Gesellschaft für Gynäkologie und Geburtshilfe

10. International Charité-Mayo-Conference -

Updates in Gynecology: A Global Perspective
Information: www-imsvancouver2018.com

Information: www.senologiekongress.de

Information: www.dgp2018.de

Information: www.ecco-org.eu

Information: www.igcs2018.com

Information: www.haematologie-onkologie-2018.com

Information: www.dggg2018.de

Information: www.breastanbul.org

\section{Information:}

www.esmo.org/Conferences/ESMO-2018-Congress

Information: www.dggg 2018 .de

Information:

www.charite-mayo.de/2017/pdf/CMC-StD-2019.pdf 